\title{
Modelo educativo emergente en las buenas prácticas TIC
}

\author{
Educational emergent model in the good practices ICT \\ María Dolores Fernández Alex \\ Centro de Magisterio Virgen de Europa. Adscrito a la Universidad de Cádiz.
}

\author{
Recibido: 19/05/2016
}

Aceptado: 23/06/2016

\begin{abstract}
Knowledge and Information Society poses new educational challenges, schools must train ditizens for the 21st century. It is not possible to develop the necessary skills from traditional approaches; we will have to tackle innovations and changes to make them possible. Information and communications technology (ICT) offers enorm ous potential for change in education. In order to realize the use of ICT that it should be made in ord er to undertake the necessary innovations, we analyse different experiences with ICT developed in primary and secondary schools. The experiences analysed were nine and they are located in seven centres in the province of Cadiz. The teachers responsible for the teaching practices were conducted narrative interviews that were analysed using the qualitative analysis programme ATLAS.ti (v. 6.2). From the data obtained, we connected the characteristics of each experience with the characteristics of what a good teaching practice with ICT would be. Afterwards, a global analysis that would allow us to find matching elements between them was carried out. From these coincidences, we outline the most common model practice amongst the analyzed experiences, a model that marks the trend to which teachers that make use of ICT approximate, identifying the direction of change from educational practice approach.
\end{abstract}

KEY WORDS: Good educational practices, educational model, educational innovation, Knowledge Society, educational methodology

\section{RESUMEN}

La Sociedad del Conocimiento y la Información plantea nuevos retos educativos, la escuela, debe capacitar a cudadanos del siglo XXI. Desde los planteamientos tradicionales no es posible desarrollar las competencias necesarias, hay que abordar innovaciones y cambios que las hagan posible. Las Tecnologías de la Información y la Comunicación (TIC) ofrecen enormes posibilidades de cambio en los procesos educativos. Con el objetivo de concretar el uso que de las TIC se debería realizar para con ellas acometer las innovaciones necesarias, analizamos distintas experiencias con TIC desarrolladas en centros de primaria y secundaria. Las experiencias analizadas fueron nueve, localizadas en siete centros de la provincia de Cádiz. A los profesores responsables de las prácticas educativas se les realizaron entrevistas narrativas que fueron analizadas con el programa de análisis cualitativo Atlas.Ti (v. 6.2). A partir de los datos obtenidos, relacionamos las características de cada experiencia con las características de lo que sería una buena práctica docente con TIC, pasando después a un análisis global que nos permitiera encontrar elementos coincidentes entre ellas. A partir de estas coincidencias, esbozamos el modelo de práctica más común entre las experiencias analizadas, modelo que nos marca la tendencia a la que se aproximan los docentes que hacen uso de las TIC, identificando así el sentido del cambio desde la práctica educativa.

PALABRAS CLAVE: Buenas prácticas docentes, TIC, Modelo Educativo, Innovación educativa, Sociedad del Conocimiento, Metodología educativa.

\footnotetext{
Dirección de correspondencia:

María Dolores Fernández Alex, Centro de Magisterio Virgen de Europa. Centro Adscrito a la, Universidad de Cádiz.e-mail: fernandez.alex@magisteriolalinea.com
} 


\section{Sociedad del Conocimiento y la Información: reto educativo}

La Sociedad del Conocimiento y la Información ofrece un mundo lleno de posibilidades, hacerlas realidad pasa por afrontar el reto de aprender a vivir en ella. Es necesario formar ciudadanos del siglo XXI y para ello, la escuela debe ofrecer procesos educativos innovadores que lleven a la mejora y al cambio educativo. La cuestión que se plantea es hacia dónde nos debemos dirigir y qué procesos se deben iniciar. En esta línea, la UNESCO (2004) también considera primordial proveer a los alumnos de los conocimientos y herramientas necesarias para el siglo XXI, su propuesta es que los sistemas educativos de todo el mundo se enfrenten al desafío de utilizar las nuevas Tecnologías de la Información y la Comunicación (TIC).

El proceso de incorporación de las TIC iniciado hasta el momento en los procesos educativos responde más a intereses puramente comerciales, tal como Gutiérrez y Tyner (2012) afirman, no llega a suponer una integración y cambio a nivel curricular. Habría que reconsiderar los objetivos y contenidos educativos que actualmente se trabajan en la escuela, tal como Coll (2007) apunta, plantear nuevas formas de alfabetización que faciliten el desarrollo de las competencias acordes a las demandas que la sociedad actual plantea, que capacite para hacer un uso crítico de TIC.

Distintos son los términos que se utilizan para hacer referencia a esta nueva alfabetización, la alfabetización del siglo XXI, todos ellos, según plantea García (2012), estarían relacionados: alfabetización digital o informática, multimedia o en medios, informacional y la multialfabetización. La alfabetización informacional es el término que González (2012) utiliza para referirse a un proceso que iría más allá de la alfabetización digital, ya que implicaría integrarse en un conjunto de alfabetizaciones, mediática, tecnológica, informática, social, crítica, etc., en beneficio de la formación del usuario en las competencias que le son básicas en la sociedad actual. Este proceso de alfabetización, para Area y Pessoa (2012), exige de un modelo educativo integral y globalizado.

Este es un desafío que se debe abordar de forma clara y prioritaria, aunque sin obviar que, tal como advierten Castillo y De Benito (2008), las transformaciones en el ámbito educativo se producen a menor velocidad que las sociales. El hecho de que las prácticas de enseñanza no vayan acorde a las innovaciones tecnológicas, no puede suponer un freno al cambio.

Valverde, Garrido y Sosa (2010) reconocen dos visiones de la pedagogía: una, más tradicional, que relacionan con la sociedad industrial; y, una segunda, más progresista, relacionada con la Sociedad del Conocimiento y la Información. La visión más tradicional otorga al profesor un papel más prescriptivo y al alumno un papel pasivo en los procesos de aprendizaje; la docencia se planifica para el gran grupo favoreciendo un aprendizaje individualizado y reproductivo, los grupos se consideran homogéneos. Por su parte, la visión más emergente le otorga protagonismo al alumno, las actividades son determinadas por este, se generan dinámicas de trabajo en equipo, el aprendizaje es productivo, el docente organiza la docencia en pequeños grupos y el trabajo se genera en equipos de profesores.

El éxito o fracaso de las innovaciones educativas depende, en gran parte, tal como González (2008) señala, de la forma en la que los diferentes actores educativos interpretan, redefinen, filtran y dan forma a los cambios propuestos. Las innovaciones en educación tienen ante sí como principal reto los procesos de adopción por parte de las personas, los grupos y las instituciones.

Desde la segunda mitad del siglo XX, tal como apuntan Aparici y Silva (2012), se viene realizando una seria crítica al modelo pedagógico transmisor caracterizado por la unidireccionalidad o una seudobidireccionalidad basada en los modelos funcionalista de comunicación. Se convierte en un reto desarrollar e implementar modelos y prácticas basadas en la interactividad, porque afecta a los principios de construcción del conocimiento, al establecimiento de nuevas formas de aprender y de enseñar.

Esteve (2009) identifica un desfase entre la potencialidad de las TIC que se han incorporado en las aulas y la escasa renovación en los procesos pedagógicos.

Por su parte, García-Valcárcel, Basilotta y López (2014) plantean como imprescindible la transformación de las prácticas educativas hacia modelos que promuevan proyectos colaborativos apoyados en el uso de las TIC, ya que facilitarán un canal de comunicación e información necesario 
en escenarios de aprendizaje abiertos, interactivos, motivadores para el alumnado y centrado en el desarrollo de competencias.

Esta transformación la hacen posible las Buenas Prácticas Pedagógicas con TIC. El poder identificar y hacer visibles estas Buenas Prácticas va a permitir, tal como Zabalza (2012) apunta, que los docentes mejorar su práctica educativa. Para alcanzarlas, el primer paso es definirlas, tarea que resulta compleja. De Pablos y González (2007) las conciben como

La actuación o conjunto de actuaciones desarrolladas en un centro escolar para facilitar procesos de integración de las TIC, sistematizadas y experimentadas que supongan un posicionamiento por parte de quién las implementa sobre el objetivo educativo que persigue y sobre el papel que juegan las TIC en la consecución del objetivo planteado. (p. 11)

Contrastando esta definición con la de otros autores, encontramos elementos coincidentes, el más destacado, el suponer una mejora en el proceso educativo, (Palomo, Ruiz y Sánchez, 2005; Marqués, 2005; De Pablos y González, 2007; De Pablos y Jiménez, 2007; González y Rodríguez, 2010); seguido de la influencia del contexto (De Pablos y González, 2007; González y Rodríguez, 2010; y Zabalza, 2012); y por último, ofrecer un modelo de actuación (De Pablos y González, 2007; Marqués 2005; Zabalza, 2012).

Para que la incorporación de las TIC suponga una Buena Práctica Pedagógica que mejore los procesos educativos, habría que tener en cuenta los principios en los que se debería apoyar dicha práctica. Chickering y Gamson (1987) y Chickering y Ehram (1996) concretan siete principios a tener en cuenta en la incorporación de las TIC en las aulas como son que estimula el contacto entre profesor y alumno, las TIC incrementan el acceso del estudiante al profesor, ayuda a los más tímidos, facilita la comunicación asincrónica; desarrolla la reciprocidad y la cooperación entre estudiantes; usa de técnicas de aprendizaje activo, las TIC aportan herramientas y recursos para aprender haciendo; proporciona un feedback inmediato; permite la realización de tareas en los tiempos previstos ya que las TIC motivan, facilitan el acceso a recursos; implica comunicar expectativas positiva a todos los alumnos; respeta la diversidad de capacidades y modos de aprendizaje, las TIC ofrece distintos modos de acercar a todos los alumnos a los contenidos.

A estos siete principios, Olalla y Primo (2006) añaden seis más: usar las TIC sin que implique una pérdida de contenidos; adaptar las TIC a la enseñanza y no al revés, incorporar las TIC sin ninguna finalidad específica puede llevar a un proceso educativo centrado en las TIC y no en el aprendizaje del alumnado; no canalizar los procesos de enseñanza-aprendizaje a través de las TIC, suponen un recurso, pero si este recurso llega a ser el único canal, se corre el riesgo de no alcanzar los objetivos propuestos; evitar el activismo tecnológico, si llegamos a perder la referencia del objetivo a alcanzar se puede correr el riesgo de quedarnos en la tarea a realizar, no llegando a alcanzar los objetivos perseguidos; no utilizar las TIC para "evadirse" de los problemas reales de la clase; la formación permanente del profesorado, acompañando el ritmo de las innovaciones educativas.

Todos los principios presentados podrían complementarse con algunas recomendaciones que Área (2007) presenta para la actuación y uso de las TIC en el aula. Un profesor o profesora debe ser consciente de que las TIC no tienen efectos mágicos sobre el aprendizaje ni generan automáticamente innovación educativa. Es el método o estrategia didáctica junto con las actividades planificadas las que promueven un tipo u otro de aprendizaje; con un método de enseñanza expositivo, las TIC refuerzan el aprendizaje por recepción, con un método de enseñanza constructivista, las TIC facilitan un proceso de aprendizaje por descubrimiento. Las TIC pueden ser utilizadas tanto como herramientas para la búsqueda, consulta y elaboración de información como para relacionarse y comunicarse con otras personas. Es decir, debemos propiciar que el alumnado desarrolle con las TIC tareas tanto de naturaleza intelectual como de interacción social. Usar las TIC no debe considerarse ni planificarse como una acción ajena o paralela al proceso de enseñanza 
habitual, es decir, las actividades de utilización de los ordenadores tienen que estar integradas y ser coherentes con los objetivos y contenidos curriculares que se están enseñando.

La postura que Durán, Estay-Niculca y Álvarez (2015) defienden es clara, la incorporación de Buenas Prácticas Educativas permite dinamizar los procesos educativos.

\section{Proceso de investigación}

El proceso de investigación que desarrollamos persigue conocer en qué medida la integración de las TIC en los procesos educativos están formando a alumnos competentes para desenvolverse en la Sociedad del Conocimiento y la Información, está posibilitando herramientas para la Sociedad del Siglo XXI. El trabajo realizado se ha centrado en los objetivos que a continuación detallamos.

a) Analizar qué modelo de enseñanza y aprendizaje apoyan las Buenas Prácticas Pedagógicas que se constaten así como relacionarlas con la concepción educativa en las que se sustentan.

b) Identificar cuáles de las prácticas localizadas en los centros de primaria y secundaria del Campo de Gibraltar se ajustan al concepto de Buenas Prácticas Docentes con TIC.

c) Reconocer las innovaciones que se han producido en los procesos de aprendizajeenseñanza tras la incorporación de las TIC en las iniciativas educativas identificadas como Buenas Prácticas Pedagógicas.

La investigación que presentamos es un estudio de caso centrado en experiencias educativas desarrolladas en las etapas de educación obligatoria de los centros del Campo de Gibraltar. Optamos por el estudio de casos porque nos va a permitir comprender el objeto de nuestro estudio desde las variables del contexto que lo hacen particular y complejo (Stake, 2005; Yin, 1989).

Tras seleccionar las experiencias educativas que formaría parte del estudio, pasamos a analizarlas para conocerlas en profundidad, así como visibilizarlas para que puedan ser referentes para los nuevos centros que se planteen la incorporación de las TIC. A los docentes responsables de dichas experiencias se les realizaron entrevistas narrativas permitiéndoles así reflexionar y compartir el proceso de incorporación de las TIC en su centro y en su aula. En estas entrevistas narran motivaciones, intenciones, decisiones, acciones... que van a posibilitar hacer presente el proceso de cada experiencia. Perseguimos constatar a través de lo narrado los cambios que la incorporación de las TIC han provocado en los procesos de enseñanza y aprendizaje, intentando identificar qué modelo de enseñanza y aprendizaje era el perseguido por cada docente e identificar las innovaciones educativas que se están desarrollando para concluir en qué medida se ajustan a una Buena Práctica con TIC.

\subsection{Muestra del estudio}

Las experiencias educativas en las que se ha centrado el estudio son experiencias desarrolladas en centros de educación primaria y secundaria del Campo de Gibraltar. La selección de los centros se hizo en colaboración con el Centro de Profesores del Campo de Gibraltar siendo el criterio de referencia que fuesen centros que estuvieran desarrollando prácticas innovadoras con TIC con cierta trayectoria en su implantación, evitando así experiencias puntuales. Este primer contacto lo tuvimos a principios del curso 2008-09. Aún no se había implantado el programa Escuela TIC 2.0, y la realidad que el coordinador TIC del CEP nos hizo llegar es que solo había un centro en la zona centros que cumplieran esas condiciones. Algunos centros estaban iniciando alguna experiencia en ese curso, pero nada consolidado, no contaban con una trayectoria en la implantación de las TIC. Este hecho nos obligó a ampliar la zona de estudio a la provincia de 
Cádiz, contactando con otros CEP de la provincia, en concreto con el CEP Cádiz y CEP Jerez de la Frontera. Siete fueron finalmente los centros que formaron parte de nuestro estudio: CEIP Erytheia (San Fernando), CEIP Ruiz Enciso (Medina Sidonia), CEIP Los Bateles (Conil de la Frontera), IES Saladillo (Algeciras), IES Caleta (Cádiz), CEIP Andalucía (Rota), IES Fernando Quiñones (Chiclana de la Frontera).

\subsection{Metodología de investigación}

Tal como hemos mencionado anteriormente, el estudio se trata de un estudio de caso en el que hemos analizado las prácticas educativas de nueve docentes de siete centros educativos distintos. A cada docente se le realizaron entrevistas narrativas en dos momentos: un primero para establecer el primer contacto; y un segundo para profundizar en alguno de los aspectos señalados. Los datos obtenidos en estas entrevistas fueron analizados con el programa de análisis cualitativo Atlas.ti (v.6.2).

La narrativa como enfoque de investigación, según Bolívar (2012), permite identificar las pautas y formas de construir sentido a partir de acciones temporales personales, por medio de la descripción y análisis de los datos biográficos. También es una reconstrucción de la experiencia, porque mediante un proceso reflexivo, se da significado a lo sucedido o vivido convirtiéndolo en una trama argumental, secuencia temporal, personajes y situación. Esta narrativa cumpliría, para Huchim y Reyes (2013), una tarea central como es la de comprender la historia de una persona. Al tratar de contar su historia en palabras, reflexionando sobre su vida y explicarlo a los demás, el texto se convierte en el elemento más importante que implica recontar e intentar revivir esa historia.

Para Bolívar, Segovia y Fernández (2001) la investigación narrativa se ha legitimado como una forma de construir conocimiento en la investigación educativa y social, un lugar de encuentro e intersección entre diversas áreas sociales, que relaciona diversos saberes, como la teoría lingüística, historia oral e historia de vida, la antropología narrativa y la psicología.

Sin querer obviar que la información obtenida nos sitúa en la realidad de cada contexto, pensamos que sería interesante contrastar la información obtenida de los distintos docentes a fin de establecer posibles coincidencias y acercarnos a un perfil común entre ellas.

\subsection{Resultados de la investigación}

Tras analizar las distintas entrevistas realizadas, de cara a poder constatar en qué medida cada una de las experiencias suponen una Buena Práctica con TIC, así como reconocer las innovaciones educativas que plantean, pasamos a detallar los elementos de Buenas Prácticas que han sido identificados por los distintos docentes entrevistados. Elementos de BBPP en relación al alumnado (ver cuadro 1), en relación al profesorado (ver cuadro 2), en relación a la metodología (ver cuadro $3)$, en relación al centro (ver cuadro 4) y en relación a los recursos (ver cuadro 5).

Cuadro 1

Elementos de BBPP en relación al alumnado

\begin{tabular}{|c|c|c|c|c|c|c|c|c|c|}
\hline \multirow{2}{*}{$\begin{array}{l}\text { CENTROS } \\
\text { EXPERIENCIAS }\end{array}$} & \multirow{2}{*}{$\begin{array}{l}\mathrm{C} 1 \\
\mathrm{E} 1\end{array}$} & \multirow{2}{*}{$\begin{array}{l}\mathrm{C} 2 \\
\mathrm{E} 2\end{array}$} & \multicolumn{2}{|c|}{$\mathrm{C} 3$} & \multirow{2}{*}{$\begin{array}{l}\mathrm{C} 4 \\
\mathrm{ES}\end{array}$} & \multirow{2}{*}{$\begin{array}{l}\text { C5 } \\
\text { E6 }\end{array}$} & \multirow{2}{*}{$\begin{array}{l}\mathrm{C} 6 \\
\mathrm{E} 7\end{array}$} & \multicolumn{2}{|l|}{$\mathrm{C7}$} \\
\hline & & & E3 & E4 & & & & E8 & E9 \\
\hline \multicolumn{10}{|l|}{$\begin{array}{l}\text { ELEMENTOS } \\
\text { ALUMNOS }\end{array}$} \\
\hline Autonomia & $x$ & & $x$ & & $x$ & $x$ & $x$ & $x$ & $x$ \\
\hline Perseverancia & & $x$ & & & & & & & \\
\hline Implica & & $x$ & & & & & & & \\
\hline Responsabilidad & $x$ & & $x$ & $x$ & & & & & \\
\hline Cooperación & & & & & $x$ & & & & \\
\hline Buen clima & & & & & & & & $x$ & \\
\hline Al investigador & $x$ & & & & & & & & \\
\hline Alumno motivado & & & $x$ & & & & & & \\
\hline Aprend activo & $x$ & & $x$ & $x$ & & & & & $x$ \\
\hline Creatividad & & & & & & & & $x$ & \\
\hline
\end{tabular}


Cuadro 2

Elementos de BBPP en relación al profesorado

\begin{tabular}{|c|c|c|c|c|c|c|c|c|c|}
\hline CENTROS & $\mathrm{Cl}$ & $\mathrm{C} 2$ & $\mathrm{C} 3$ & & $\mathrm{C}_{4}$ & CS & C6 & & $\mathrm{C7}$ \\
\hline EXPERIENCIAS & E1 & E2 & E3 & E4 & E5 & E6 & E7 & E8 & E9 \\
\hline \multicolumn{10}{|l|}{$\begin{array}{l}\text { ELEMENTOS } \\
\text { PROFESORADO }\end{array}$} \\
\hline Cambio cultura docente & & $x$ & & & & & & & \\
\hline Trabajo en equipo/cooperat. & $x$ & $\mathrm{x}$ & $x$ & $\mathrm{x}$ & $x$ & $\mathrm{x}$ & $x$ & $x$ & $x$ \\
\hline Nuevo rol facilitador & $x$ & & $x$ & & $x$ & & $x$ & & \\
\hline Motivación del profesorado & & & & $x$ & & $x$ & & $x$ & \\
\hline Investigación uso tic & & $x$ & & & & & & & \\
\hline Elaboración material/ rec & $x$ & & $x$ & & $x$ & & & $x$ & \\
\hline Compartir material/rec & $x$ & & $x$ & & & $x$ & & $x$ & \\
\hline Formación continuáperman. & & & $\mathrm{x}$ & $\mathrm{x}$ & $\mathrm{x}$ & & $\mathrm{x}$ & & \\
\hline \multicolumn{10}{|l|}{ Iniciativa } \\
\hline Accesibilidad & & & & & & & & & \\
\hline
\end{tabular}

Cuadro 3

Elementos de BBPP en relación a la metodología

\begin{tabular}{|c|c|c|c|c|c|c|c|c|c|}
\hline CENTROS & $\mathrm{Cl}$ & $\mathrm{C} 2$ & $\mathrm{C} 3$ & & $\mathrm{C} 4$ & C5 & C6 & & C7 \\
\hline EXPERIENCIAS & E1 & E2 & E3 & E4 & ES & E6 & E7 & E8 & E9 \\
\hline \multicolumn{10}{|l|}{$\begin{array}{l}\text { ELEMENTOS } \\
\text { METODOLOGIA }\end{array}$} \\
\hline Aprendizaje autónomo & $\mathrm{x} \times$ & & & & & $\mathrm{x}$ & & & \\
\hline Aprendizaje funcional & 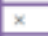 & $x$ & & & & & & & \\
\hline Aprendizaje contextualizado & $x$ & & $x$ & $x$ & & & & & $x$ \\
\hline Aprendizaje sipnificativo & & $x$ & & & & & & & \\
\hline Aprendizije coopentivo & & $x$ & & & & & & & \\
\hline Autoaprendizaje & & $x$ & & & & & & & \\
\hline Seguimiento del alumno & & & & & $x$ & & & & \\
\hline Aprend compet vivir/sociedad & & & & $\bar{x}$ & & & & & \\
\hline Nuevos proc. Metodolópicos & & & & & $x$ & & 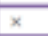 & & $\bar{x}$ \\
\hline Flexubulizar procesos & & & & & & & & & $x$ \\
\hline Enseñanza centrada & & & & & $\bar{x}$ & & & & \\
\hline Evaluación proceso & & & & & $x$ & & & & \\
\hline Mejores resultados & & & & & $\bar{x}$ & & & & \\
\hline Motivación & & & & & $\bar{x}$ & & & & \\
\hline Atención a la daversidad & & & $x$ & $x$ & & & $x$ & $x$ & \\
\hline \multicolumn{10}{|l|}{ Tic al servicio de th ensetian } \\
\hline Interdisciplinariedad & & & & & & & & & \\
\hline Variedad recursos & & & & & & & & & \\
\hline
\end{tabular}

Cuadro 4

Elementos de BBPP en relación al centro

\begin{tabular}{|c|c|c|c|c|c|c|c|c|c|}
\hline CENTROS & $\mathrm{Cl}$ & $\mathrm{C} 2$ & $\mathrm{C} 3$ & & $\mathrm{C}_{4}$ & C5 & $\mathrm{C} 6$ & & C7 \\
\hline EXPERIENCLAS & E1 & E2 & E3 & E4 & ES & E6 & E7 & E8 & E9 \\
\hline \multicolumn{10}{|l|}{$\begin{array}{l}\text { ELEMIENTOS } \\
\text { CENTROS }\end{array}$} \\
\hline Apoyo equipo directivo & $x$ & & & & & & & & \\
\hline Apoyo tie & & & & & & $x$ & $x$ & $x$ & $x$ \\
\hline Coordenación & & & & $x$ & & & & & \\
\hline Conmpartir experiencias & & $x$ & & & & & & & \\
\hline Autonomia del centro & & $x$ & & & & & & & \\
\hline Vinculación entre centro & & $x$ & & & & & & & \\
\hline Oferta de formación & & & & & & $x$ & & & \\
\hline Grupos de trabajo & & & & & & & $x$ & $x$ & $x$ \\
\hline \multicolumn{10}{|l|}{ Cultura centro } \\
\hline \multicolumn{10}{|l|}{ Experiencia previa } \\
\hline \multicolumn{10}{|l|}{ Detención y difusión } \\
\hline \multicolumn{10}{|l|}{ Impacto } \\
\hline Sostenibilidad & & & & & & & & & \\
\hline
\end{tabular}

Revista Fuentes

2016, 18 (1), 33-47 
Cuadro 5

Elementos de BBPP en relación a los recursos

\begin{tabular}{|c|c|c|c|c|c|c|c|c|c|}
\hline \multirow{2}{*}{$\begin{array}{l}\text { CENTROS } \\
\text { EXPERIENCIAS }\end{array}$} & \multirow{2}{*}{$\begin{array}{l}\mathrm{C} 1 \\
\mathrm{E} 1 \\
\end{array}$} & \multirow{2}{*}{$\begin{array}{l}\mathrm{C} 2 \\
\mathrm{E} 2 \\
\end{array}$} & \multicolumn{2}{|l|}{$\mathrm{C} 3$} & \multirow{2}{*}{\begin{tabular}{|l} 
C4 \\
E5 \\
\end{tabular}} & \multirow{2}{*}{$\begin{array}{l}\text { C5 } \\
\text { E6 }\end{array}$} & \multicolumn{2}{|l|}{$\mathrm{C} 6$} & \multirow{2}{*}{$\begin{array}{l}\text { C7 } \\
\text { E9 }\end{array}$} \\
\hline & & & E3 & E4 & & & E7 & E8 & \\
\hline \multicolumn{10}{|l|}{$\begin{array}{l}\text { ELEMENTOS } \\
\text { RECURSOS }\end{array}$} \\
\hline Elaboración material & $x$ & & $x$ & $\mathrm{x}$ & & & & $x$ & $x$ \\
\hline Compartir materiales & $x$ & $x$ & & & & & & & $x$ \\
\hline \multicolumn{10}{|l|}{ Reutilizar } \\
\hline \multicolumn{10}{|l|}{ Racionalizar } \\
\hline Adaptar recursos & & & $\mathrm{x}$ & & & & & & \\
\hline Recursos diversidad & & $\mathrm{x}$ & & $x$ & $\mathrm{x}$ & $x$ & $\mathrm{x}$ & $x$ & $x$ \\
\hline Red de ordenación & & $\mathrm{x}$ & & & & & & & \\
\hline
\end{tabular}

En relación al alumnado, el perfil de alumno que encontramos en estas prácticas educativas es el de un alumno motivado con el uso de las TIC, porque las reconoce como facilitadoras del aprendizaje, y que las utiliza para convertirse en un alumno que se implica en los procesos de aprendizaje (Marqués, 2005), participativo y activo, y que le llevan a ser un alumno investigador, responsable de su aprendizaje donde elabora sus propios materiales y recursos (Gallego, 2011; Casanova y Pavón, 2010). No se identifica a ningún alumno que el uso de las TIC le haga ser más perseverante y creativo (Marqués, 2005). Tampoco identificamos a un alumnado que comparta esos materiales que elabora y con los que trabaja (Casanova y Pavón, 2010).

Como elemento emanado del análisis, encontramos que las TIC han favorecido procesos de aprendizaje cooperativos y que han generado un buen clima de clase.

El perfil docente que encontramos es un docente motivado, que trabaja en equipo y de manera cooperativa (Marqués, 2005; DECOM 2009; Casanova y Pavón, 2010). Uno de los centro ha creado un grupo de trabajo con TIC que apoya el estudio e implantación de las TIC en el aula. De este modo, un docente con un nuevo rol, se convierte en facilitador y mediador de los aprendizajes, con un alumnado más accesible al que se le puede realizar un mayor seguimiento con ideas que se aproxima a un nuevo modelo cultural (Dulac, 2004; Marqués, 2005; Valverde, 2011). Por lo tanto, son docentes con disposición a la formación permanente como necesidad de cara a la innovación (Dulac, 2004). Se convierte así, en un docente que no solo selecciona los medios y recursos existentes, sino que también elabora sus propios materiales y recursos (Dulac, 2004). No solo se conforman con crear los materiales, sino que los comparten con compañeros del centro y de otros centros, creándose así relaciones de trabajos con otros. Por ello, coinciden en impulsar en mayor medida el uso de las TIC, para así convertirse en el motor del cambio del centro. Valverde, (2011) señala un aspecto que no ha sido destacado como es que el uso de las TIC genere altas expectativas en el profesorado sobre el aprendizaje del alumnado.

En relación a la metodología, uno de los aspectos marcados por los docentes es la posibilidad de atender a la diversidad, de permitir ajustar a la enseñanza los ritmos y necesidades de los alumnos. Y así, de este modo, poder flexibilizar los procesos de aprendizaje y los contenidos, y diversificar las actividades a partir de procesos de evaluación continua (Marqués, 2005; Gómez, 2011; y, DECOM, 2009). Como consecuencia, posibilitan los procesos de enseñanza centrada en la vida del alumno y para la vida (DECOM, 2009).

Esta metodología está enfocada a conseguir un aprendizaje autónomo, funcional, contextualizado, un aprendizaje significativo, aplicado y que fomenta el autoaprendizaje. Esto permite que se generen nuevos procesos de enseñanza, que facilitan el contacto y seguimiento del alumno. 
Continuando con la metodología, dos son los aspectos señalados desde los autores y no identificados en las prácticas analizadas, como son: aprender de forma interdisciplinar (Gallego, 2011); y, favorecer el aprendizaje crítico (Valverde, 2001).

Los centros se caracterizan por contar con gran apoyo por parte del equipo directivo, así como por parte del coordinador TIC (De Pablos y González, 2007). La cultura que se identifica es de trabajo coordinado de profesorado, ya que crean grupos de trabajo, en las que se comparten las experiencias TIC y se establecen relaciones con otros centros (Cebrián de la Serna, 2011; DECOM, 2009; y, De Pablos y González, 2007). Se considera que la formación del profesorado es una necesidad detectada por los centros de cara a la implantación de las TIC (Dulac, 2004; DECOM, 2009).

Todos los centros que han iniciado el trabajo con TIC siguen desarrollando la experiencia, sin plantearse, a pesar de las posibles dificultades, abandonar el proyecto (De Pablos y González, 2007).

A pesar de esto, algunos aspectos señalados desde la teoría no han sido identificados en las prácticas analizadas como son la autonomía de los centros de cara a la flexibilidad curricular (Comisión Europea Education and Training, 2003), y la detección y difusión de las Buenas Prácticas, (Azpetia, Monge y Ovelar, 2005; y, MEC, 2004), por lo tanto, al ser una actividad que no se ha identificado en las prácticas, sería un aspecto a potenciar.

Aunque algún centro ha nombrado que las TIC han favorecido la relación con otros centros, no es una práctica común (De Pablos, 2012; DECOM, 2009; De Pablos y González, 2007; y, Marqués, 2005), por lo que sería necesario reforzar redes de apoyo y participación y llegar a ser comunidades de aprendizaje.

Por otra parte, algunos aspectos a identificar desde una de las prácticas analizadas es la nueva relación que el uso de las TIC favorece entre el centro y la administración. Así como, otro aspecto no recogido hasta el momento, en otra de las prácticas, es la creación en el centro de la figura del tutor TIC para responder de mejor manera al seguimiento, orientación y apoyo a los docentes en la implantación de las TIC. Esta acción la hizo posible la motivación e implicación del profesorado del centro. Un último aspecto sería el trabajo con las familias, ya que la mayoría de los centros señalan el trabajo con las familias como un elemento clave. Consideraban que es necesario que los padres sean conscientes de la posibilidad de que las TIC ofrece, esto ha llevado a que desde los centros se responda a esta necesidad ofreciendo jornadas formativas.

Para terminar, se constata una gran variedad en los recursos utilizados. Los profesores entrevistados han elaborado sus recursos y materiales, y muchos de ellos los han compartido (Area, San Nicolás y Fariña, 2010; MEC, 2004), aunque habría que difundirlos más de lo que lo hacen. Reconocen que reutilizan los materiales, al ponerlos a disposición de sus compañeros (Azepetia, Monge y Ovelar, 2005).

Por lo tanto, se considera importante destacar que, la finalidad del uso de las TIC es educativa y su uso debe ser como herramientas de enseñanza y aprendizaje y no como mera finalidad (DECOM, 2009).

Relacionando los aspectos identificados con las recomendaciones de Area, (2007), podemos decir que sí evidencian su preocupación porque reconocen que lo importante es lo educativo y las TIC solo son herramientas que buscan con su uso en el aula un aprendizaje activo y responsable por parte del alumno, es decir, pretenden buscar un aprendizaje cooperativo. Las TIC están integradas en el desarrollo de las distintas materias, destacando solo un centro la formación específica en TIC acorde a las finalidades del centro.

Tomando como referencia el paradigma desde el que se enfocan la mayoría de actividades y tareas descritas, dos de ellas se enmarcarían en el paradigma conductual, como son la Experiencia 4 (CEIP Los Bateles) y la Experiencia 8 (CEIP Andalucía); otros dos se situarían en el paradigma cognitivo, como son Experiencia 3 (CEIP Los Bateles), Experiencia 5 (IES Saladillo); y, tres de ellas en el paradigma ecológico, Experiencia 1 (CEIP Erytheia), Experiencia 2 (CEIP Ruiz Enciso) y Experiencia 6 (IES Caleta). Las dos experiencias restantes, la Experiencia 7 (CEIP Andalucía) y la Experiencia 9 (IES Fernando Quiñones) son difíciles de enmarcar, ya que comparten de manera 
equilibrada características de dos paradigmas, podríamos decir que están situadas en el paradigma cognitivo en evolución al paradigma ecológico. Constatamos, por tanto, que el mayor peso lo tienen las experiencias ecológicas (paradigma centrado en la vida y en el contexto) siendo lo que el alumno vive lo que provoca los procesos de aprendizaje. En este sentido, las necesidades formativas del alumno respecto a la sociedad que vive estarían cubiertas. No podemos olvidar que seguimos identificando modelos desde paradigmas más desfasados en el tiempo pero la tendencia mayor se sitúa en paradigmas más actuales.

Los modelos y enfoques de enseñanza identificados son los que plantean una línea de trab ajo coherente con los paradigmas en los que han sido enmarcados. Es decir, las experiencias situadas en el paradigma conductual se identifica con un modelo de enseñanza de proceso-producto y en un enfoque centrado en la transmisión cultural. El modelo de enseñanza, es mediacional en las experiencias situadas en el cognitivo, y ecológica en las experiencias del paradigma ecológicocontextual. En relación a los enfoques de enseñanza no encontramos una clara diferenciación, y es que, tanto los que se sitúan en el paradigma cognitivo como en el ecológico o en proceso entre uno y otro, identificamos enfoques que por momentos se centran en el entrenamiento de habilidades, $o$ en el fomento del desarrollo natural o en la producción de cambios conceptuales. En las experiencias ecológicas, identificamos una preocupación por el contexto, así como por promover en las aulas un alumnado activo y responsable.

\section{Conclusiones}

Tras conocer y analizar distintas experiencias desarrolladas en centros de primaria y secundaria de la provincia de Cádiz, podemos afirmar que tal como apuntábamos con anterioridad, cada experiencia es única y singular por los aspectos del contexto que la determinan y por el planteamiento educativo desde el que cada docente desarrolla sus clases. Es este último punto el que nos interesa conocer y analizar: los modelos de enseñanza y aprendizaje que cada uno desarrolla con el apoyo de las TIC. Por eso, siguiendo la clasificación de los paradigmas educativos realizada, encontramos un elemento común en todas ellas, y es que ninguna de las experiencias refleja características de un único paradigma, sino de distintos que se complementan. El planteamiento es coherente en todos los casos porque supone enfocar las actividades desde distintas perspectivas y, de esta forma, se enriquecen los procesos.

Ninguna de las experiencias se ajusta a las características que definen los paradigmas y modelos educativos, aunque sí que identificamos características más próximas a uno de ellos. Siguiendo la clasificación de Román Pérez (1990), dos de las experiencias se podrían enmarcar en el paradigma competencial, tres en el paradigma ecológico, dos en el paradigma mediacional. Las dos restantes las situamos entre el paradigma mediacional y el ecológico, ya que vemos que el docente en ocasiones asume el rol de mediador y en otras de gestor.

En relación a los modelos de enseñanza de Pérez Gómez (2000), identificamos que dos centros claramente siguen el modelo relacional, otros dos se sitúan en el de proceso-producto, y el resto en el ecológico o con la intención de llegar a serlo.

Independientemente del paradigma o modelo en el que sitúen, encontramos dos aspectos coincidentes entre los distintos docentes: uno, reconocen las TIC como herramientas que ofrecen la posibilidad de cambiar los procesos; y, dos, que las TIC permiten flexibilizar los procesos educativos y facilitan así la atención a la diversidad de los alumnos.

En relación al "nuevo modelo de escuela", hemos tenido como referencia el modelo que presentamos en el cuadro 6, en el que diferenciamos aspectos característicos en relación al alumnado, profesorado, aprendizaje y enseñanza. Podemos constatar que el uso de las TIC en el aula ha provocado cambios en el alumnado, en el aprendizaje, en el profesor y/o en la enseñanza respecto al modelo tradicional en todas las experiencias.

- En relación al alumnado, encontramos en la mayoría de experiencias un nuevo rol de alumno, ya que pasa a ser un alumno autónomo en sus procesos de aprendizaje, activo en todos los procesos que se desarrollen y constructor de su aprendizaje. Y al mismo tiempo, 
asume las tareas como responsable de las mismas, es decir, su aprendizaje va a depender de sus decisiones y acciones.

- En relación al aprendizaje, identificamos experiencias en las que el aprendizaje es contextualizado, parte de la vida del alumno y/o la integra en los procesos. En la mayoría de los casos, el aprendizaje se desarrolla de manera social y compartida, dirigido por el propio alumno, que, como antes dijimos, es responsable de su aprendizaje pero con otros y junto a otros, de manera colaborativa.

- En relación al profesorado, identificamos un nuevo rol docente como elemento de innovación. Las experiencias nos evidencian docentes preocupados por ser guía en el proceso de aprendizaje, pasando de ser transmisor en el aula a ser facilitador del proceso.

- En relación a la nueva enseñanza, todas las experiencias coinciden en hacer un uso de las TIC con fines pedagógicos, superando su uso como un mero recurso en el aula. Las TIC posibilitan a los docentes que la actividad del alumno sea la base en la construcción del conocimiento. También se convierten en herramientas que hacen posible la atención a la diversidad en los procesos de enseñanza-aprendizaje.

Cuadro 6

Elementos del "nuevo modelo de escuela"

\begin{tabular}{|c|c|}
\hline \multicolumn{2}{|c|}{ NUEVA ESCUELA } \\
\hline ALUMNO & APRENDIZAJE \\
\hline Autónomos & Comprensión conceptual \\
\hline Competentes & Análisis y reelaboración \\
\hline Críticos & Proceso natural \\
\hline Responsables & Contextualizado \\
\hline Constructores de conocimiento & Constructivo \\
\hline Reflexivos & Social/compartido \\
\hline Toman decisiones & Autodingido \\
\hline Creativos & Autocontrolado \\
\hline \multirow[t]{2}{*}{ Colaboradores } & Colaborativo \\
\hline & Comunidades de aprendizaje \\
\hline PROFESOR & ENSEÑANZA \\
\hline \multicolumn{2}{|l|}{ Tutor } \\
\hline Dinamizador del aprendizaje & Centrada en el aprendizaje \\
\hline Guía & Flexible \\
\hline Trabajo colaborativo & En colaboración \\
\hline Creador de ambientes & docente-alumino \\
\hline aprendizaje & Prima la actividad y Construcción \\
\hline Facilitadores de aprendizaje & del conocimiento \\
\hline Creativo & Uso pedagógico de las TIC \\
\hline \multicolumn{2}{|l|}{ Innovador } \\
\hline Competente & \\
\hline
\end{tabular}

Estas son las innovaciones que de manera más repetida hemos constado en las distintas experiencias de los centros estudiados. Hemos encontrado también elementos del nuevo modelo de escuela acorde a la sociedad que vivimos, pero no todos, ya que hay aspectos que no hemos identificado en las experiencias y que marcan el camino a seguir en este proceso de innovación con el uso de las TIC. Pasamos a concretarlos. 
- En relación al alumno, los aspectos a alcanzar serían la búsqueda de alumnos críticos, reflexivos y creativos.

- En relación al proceso de aprendizaje, aproximarnos más a alcanzar un proceso más natural en el que los conceptos son claves construidos desde la comprensión, el análisis y la reelaboración. Como elemento más clave, cabría destacar un aprendizaje que se produce en comunidad, es decir, en la que todos están implicados y todos forman parte.

- En relación al profesorado, constatar que ninguno de ellos se identifica como docente innovador, y sería positivo que lo fueran para que, a su vez, pudieran asumir el papel de innovadores de sus centros. Un punto a alcanzar sería el que fuesen docentes más preocupados por la creación de los ambientes de aprendizaje, que hemos podido ver pero no de manera mayoritaria entre los docentes entrevistados.

- Y por último, en relación a la enseñanza, dar el paso definitivo a centrarse en el aprendizaje de los alumnos y no en su enseñanza, y en la medida de lo posible, poder generar procesos de colaboración entre ellos y los alumnos.

En un segundo lugar, pasamos a concluir si las experiencias estudiadas se podrían identificar como buenas prácticas docentes con TIC, si se ajustan al concepto de Buenas Prácticas Pedagógicas centrándonos en el alumnado, en los docentes y en la metodología de enseñanza y aprendizaje que se desarrolla en cada una de ellas. Las conclusiones a las que llegamos son las siguientes.

- En relación alumnado, el perfil de alumno que encontramos en estas prácticas educativas es el de un alumno motivado con el uso de las TIC y que las reconocen como facilitadoras del aprendizaje. Se observa a un alumno que se implica en los procesos de aprendizaje, de manera participativa y activa, y que le llevan a ser un alumno investigador, responsable de su aprendizaje y que elabora sus propios materiales y recursos. A pesar de ello, no identificamos a un alumno que el uso de las TIC le haga ser más perseverante y creativo y tampoco genera dinámicas para compartir los materiales que elabora con los que trabaja.

- El perfil docente que encontramos tras el análisis es un docente motivado, que trabaja en equipo y de manera cooperativa. En algunos de los centros, esta dinámica de trabajo ha promovido la creación de un grupo que ha centrado su trabajo en la implantación de las TIC en el aula. Un docente con un nuevo rol se convierte en facilitador y mediador de los aprendizajes con un alumnado más accesible al que se le puede realizar un mayor seguimiento, por lo que se traduce en ideas que se aproximan a un nuevo modelo cultural. Se consideran docentes con disposición a la formación permanente como necesidad de cara a la innovación. Es un docente que no solo selecciona los medios y recursos existentes, sino que también elabora sus propios materiales y recursos. No les basta solo con crear los materiales, sino que los comparten con compañeros del centro y de otros centros, creándose así relaciones de trabajo con otros. De este modo, coinciden en impulsar en mayor medida el uso de las TIC y se convierten en el motor del cambio del centro.

- En relación a la metodología, uno de los aspectos señalados por los docentes es la posibilidad de atender a la diversidad y de ajustar la enseñanza a los ritmos y necesidades de los alumnos. Con esto se consigue flexibilizar los procesos de aprendizaje, los contenidos, así como diversificar las actividades a partir de procesos de evaluación continua. De esta forma, posibilitan que los procesos de enseñanza se centren en la vida del alumno y se constituyan en aprendizajes para la vida. La metodología está enfocada a conseguir un aprendizaje autónomo funcional, un aprendizaje contextualizado, un aprendizaje significativo, aprendiraje aplicado; todo esto estimula lo el autoaprendizaje. Así mismo, se generan nuevos procesos de enseñanza, que facilitan el contacto y seguimiento del alumno. También apuntan que las TIC han favorecido procesos de aprendizaje cooperativos y que han 
generado un buen clima de clase. Por otro lado, dos aspectos no señalados por los docentes, pero sí identificados como elemento de Buena Práctica serían favorecer el aprendizaje interdisciplinar y el aprendizaje crítico.

Los centros se caracterizan por contar con gran apoyo por parte del equipo directivo, así como por parte del coordinador TIC. La cultura que se identifica es de trabajo coordinado de profesorado, que crean grupos de trabajo, comparten las experiencias TIC y se vinculan con otros centros. La formación del profesorado es una necesidad detectada por los centros para favorecer la implantación de las TIC. Todos los centros que han iniciado el trabajo con TIC siguen desarrollando la experiencia, sin plantearse a pesar de las posibles dificultades, abandonar el proyecto. Los aspectos que no identificamos en relación a una Buena Práctica son la autonomía de los centros en relación con la flexibilidad curricular y la detección y difusión de las Buenas Prácticas. Aunque algún centro ha nombrado que las TIC han favorecido la relación con otros centros, no es una práctica común, por lo que sería necesario reforzar redes de apoyo y participación para que los centros lleguen a ser comunidades de aprendizaje.

En sentido contrario, encontramos también en lo narrado por los docentes aspectos que no se recogen en el modelo de Buena Práctica que hemos presentado, pero que las experiencias nos lo muestran y son la nueva relación que el uso de las TIC establece entre el centro y la administración y la creación en el centro de la figura del tutor TIC para realizar de mejor manera el seguimiento y orientación y apoyo a los docentes en la implantación de las TIC, labor que facilitó la motivación e implicación del profesorado. Un último aspecto sería el trabajo con las familias. La mayoría de los centros señalan el trabajo con las familias con un elemento clave, porque es necesario que los padres sean conscientes de las posibilidades que las TIC ofrecen; esto ha llevado a que desde los centros se responda a esta necesidad ofreciendo jornadas formativas, idea esta que coincide con la propuesta de Pérez Gómez y Sola (2010) de crear escuelas de padres para la formación en el uso y posibilidades de las TIC.

Para terminar, constatamos una gran variedad en los recursos utilizados. Los docentes han elaborado sus propios materiales, los han compartido con sus compañeros dándoles una mayor difusión, porque consideran necesario dar a conocer sus experiencias.

Relacionando los aspectos identificados con las recomendaciones de Area (2007), podemos decir que sí evidencian su preocupación porque lo importante es lo educativo. Las TIC son herramientas que buscan con su uso en el aula un aprendizaje activo y responsable por parte del alumno, es decir, un aprendizaje cooperativo. Las TIC están integradas en el desarrollo de las distintas materias, destacando solo un centro la formación específica en TIC y que se utilizan acorde a las finalidades.

De manera global, podemos afirmar que el conjunto de prácticas analizadas cumplen con los principios de una Buena Práctica señalados por Chickering y Gamson (1987) y Chickering y Ehram (1996), ya que estas prácticas han facilitado el contacto y seguimiento del alumno, han favorecido el aprendizaje cooperativo entre los alumnos generando un buen clima de clase, fomentando también el aprendizaje activo, con un mayor seguimiento de las tareas del alumno y permitiendo atender la diversidad de capacidades y modos de aprendizaje. Por consiguiente, con esto se ha conseguido que el alumno y docente hayan estado más motivados. Los docentes no han hecho alusión ni a si se realizan las tareas en los tiempos previstos ni que las TIC hayan implicado la comunicación de expectativas positivas al alumno.

En relación a los principios de Olalla y Primo (2006), los docentes entrevistados no señalan que el uso de las TIC en el aula implique pérdida de contenidos, ni que las utilicen como modo de evasión de los problemas del aula, pero sí que reconocen las TIC como herramientas, y no como una finalidad, por lo que son las TIC las que deben adaptarse a la enseñanza. Es decir, no utilizan las TIC como único recurso sino que hacen uso de recursos variados en el aula, adaptados estos siempre a las necesidades de los alumnos. Así mismo, plantean como necesario la formación permanente del profesorado para hacer un buen uso de las TIC. 


\section{Referencias bibliográficas}

Area, M. (2007). Algunos principios para el desarrollo de buenas prácticas pedagógicas con las TIC en el aula. Comunicación y pedagogía, 222, 42-47.

Area M. \& Pessoa, M.T.R. (2012). De lo sólido a lo líquido, las nuevas alfabetizaciones ante los cambios culturales de la Web 2.0. Comunicar: Revista cientfifica iberoamericana de comunicación y educación, 38, 13-20.

Area, M., San Nicolás Santos, M $M^{a}$ B., \& Fariña Vargas, E. (2010). Buenas prácticas de aulas virtuales en la docencia universitaria presencial. En J. de Pablos Pons (coord.), Buenas prácticas de enseñanza con TIC. [monográfico en línea]. Teoría de la Educación: Educación y Cultura en la Sociedad de la Información, 11(1), 7-31. Recuperado en http:/ / goo.gl/HZndvX

Azpetia, I, Monge, S \& Ovelar, R. (2005). Una aproximación al diseño de una guía de buenas prácticas en torno al paradigma de los Learning Objects. Recuperado en http://www.uoc.edu/syposia/spdece05/pdf/ID14.pdf

Bolivar, A. (2012). Metodología de la investigación biográfico-narrativa: Recogida y análisis de datos. En M.H. Abrahao (org.), Pesquisa (auto)biográfica: lugares, trajetos e desafios. V Congresso Internacional de Pesquisa (Auto)Biográfica (V CIPA). Porto Alegre: PUCRS, vol. 2.

Bolívar, A., Segovia, D., \& Fernández, M. (2001). La investigación biográfico-narrativa en educación. Enfoque y metodología. Madrid: Muralla.

Casanova, J. \& Pavón, F. (2010). Las TIC en los centros de educación obligatoria: hacia las comunidades de aprendizaje. Revista Fuentes, 10,124-139.

Castillo, P \& De Benito, B (2008). Cambios, novedades y procesos de innovación. En J. Salinas (coord), Innovación educativa y uso de las TIC (pp. 31-42). Sevilla: Universidad Internacional de Andalucía.

Cebrián de la Serna, M. (2011). Los centros educativos en la sociedad de la información y el conocimiento. En M. Cebrián de la Serna y M.J. Gallego Arrufat (coords.), Procesos educativos con TIC en la sociedad del conocimiento (pp. 23-32). Madrid: Pirámide.

Chickering, A.W \& Ehrman, S.C. (1996). Implementing the seven principles: technology as a lever. Recuperado de www.tltgroup.org/programs/seven.html

Chickering A. W. \& Gamson Z. (1987). Seven Principles for Good Practise in Undergraduate Education. American Association for Higher Education Bulletin (march): Washington, DC.

Coll, C. (2007). El bàsic imprescindible i el bàsic desitjable: un eix per a la presa de decisions curriculars en l'educació básica. En C. Coll (dir) Currículum i ciutadania El què i el per a què de l'educació escolar (pp. 227-247). Barcelona: Fundación Jaume Bofill

Comisión Europea: Education and Training (2003). Programa para la integración efectiva de las TIC en los sistemas de educación y formación en Europa. Recuperado de http://goo.gl/ 7rHG9c

De Pablos, J. (2010). Políticas educativas y la integración de las TIC a través de buenas prácticas docentes. En J. de Pablos, M. Area, J. Valverde \& J.M. Correa (coords.), Politicas educativas y buenas prácticas con TIC (pp. 21-41). Barcelona: Graó

De Pablos, J., Colás, P., González, T., \& Conde-Jiménez, J. (2015). El programa Escuela TIC 2.0 y sus efectos, según el profesorado. En J. De Pablos (coord.), Los centros educativos ante el desafío de las tecnologias digitales (pp. 85-118). Madrid: La Muralla.

De Pablos, J., Colás, P, \& González, T. (2010). Factores facilitadores de la innovación con TIC en los centros escolares. Un análisis comparativo entre diferentes políticas educativas autonómicas. Revista de Educación, 352, 23-51.

De Pablos, J., \& González, T. (2007). Politicas educativas e innovación educativa apoyada en TIC: sus desarrollos en el ámbito autonómico. Recuperado de http://goo.gl/oIo093

De Pablos, J., \& Jiménez Cortés, R. (2007). Buenas prácticas con TIC apoyadas en las Políticas Educativas: claves conceptuales y derivaciones para la formación en competencias ECTS, Revista Latinoamericana de Tecnología Educativa, 6 (2), 15-28. Recuperado de http:// campusvirtual.unex.es/cala/editio/ 
Departamento de Computación TIC Decom (2009). Manual de Buenas Prácticas en el Uso de las Tecnologías de la Información (TIC) en la Academia. Recuperado de http:/ / goo.gl/O4bDvo

Dulac, J. (2004). Referencial de buenas prácticas para el uso de las TIC en los centros educativos. Recuperado de http://goo.gl/OMa9Zi

Esteve, F (2009). Bolonia y las TIC: de la docencia 1.0 al aprendizaje 2.0. La Cuestión Universitaria, 5, 59-68.

Gallego, M.J. (2011). La integración de las tecnologías de la información y la comunicación en los centros educativos. En M. Cebrián de la Sernay M.J. Gallego Arrufat (coords.), Procesos educativos con TIC en la sociedad del conocimiento (pp. 33-44). Madrid: Pirámide.

García, J.L. (2012). Tratamiento de la información y competencia digital. En M. Díaz (coord.), Aulas del siglo XXI: retos educativos (pp. 6-39). Madrid: Ministerio de Educación, Cultura y Deporte.

García-Valcárcel, A., Basilotta, V., \& López, C (2014). Las TIC en el aprendizaje colaborativo en el aula de Primaria y Secundaria. Comunicar, 42 (XXI), 65-74.

González, N. (2012). Alfabetización para una cultura social, digital, mediática y en red. Revista Española de Documentación Cientifica, 35, 17-45.

González, J.C. (2008). TIC y la transformación de la práctica educativa en el contexto de las sociedades del conocimiento. Revista de Universidad y Sociedad del Conocimiento, 5 (2), 1-8.

González, T. \& Rodríguez, M. (2010). El valor añadido de las buenas prácticas con TIC en los centros educativos. En J. De Pablos Pons (coord.), Buenas prácticas de enseñanza con TIC [monográfico en línea]. Revista Electrónica Teoría de la Educación: Educación y Cultura en la Sociedad de la Información, 11 (1), 262-282. Recuperado de http://goo.gl/9VWBdi

Gutiérrez, A \& Tyner, K. (2012). Educación para los medios, alfabetización mediática y competencia digital. Comunicar, 38, 31-39.

Huchim, D, \& Reyes, R. (2013). La investigación biográfico-narrativa, una alternativa para el estudio de los docentes. Revista Electrónica Actualidades Investigativas en Educación, 13 (3), 1-27. Recuperado de http:/ / goo.gl/2lvKde

Marquès, P. (2005). La integración de las TIC en la escuela: las claves del éxito. Revista Comunicación y Pedagogía, 204, 37-45.

Ministerio de Educación, Cultura y Deportes (2004). Referencial de buenas prácticas para el uso de las TICs en los centros educativos. Recuperado en http://goo.gl/kI9hS6

Olalla, M.S. \& Primo, D. (2006). El perfil del maestro TIC. Recuperado de http://www.aulablog.com/files/profesor tic.ppt

Palomo, R., Ruíz, J, \& Sánchez, J. (2006). Las TIC como agente de innovación educativa. Sevilla: Consejería de Educación de la Junta de Andalucía. Recuperado de http://goo.gl/92K5ND

Pérez Gómez, A.I. (2010). Aprender a educar. Nuevos desafíos para la formación de docentes. Revista Interuniversitaria de Formación del Profesorado, 68, 37-60.

Pérez Gómez, A.I. (2010). Nuevas exigencias y escenarios para la profesión docente en la era de la información y de la incertidumbre. Revista Interuniversitaria de Formación del Profesorado, 68, 17 36.

Román Pérez, M. (1990). Curriculum y aprendizaje. $2^{\mathrm{a}} \mathrm{ed}$. Navarra; Itaka.

Stake, R. E. (2005). Investigación con estudio de casos. Madrid, Morata

UNESO (2004). Las tecnologías de la información y la comunicación en la formación docente. París: Informe UNESCO.

Valverde, J. (2011). Profesorado, Tecnología Educativa e Innovación Didáctica. En J. Valverde (coord.) Docentes E-Competentes. Buenas prácticas educativas con TIC (pp. 13-27). Barcelona: Octaedro.

Yin, R. (1989). Case Study Research. Design and Methods. London, SAGE.

Zabalza, M.A. (2012). El estudio de las buenas prácticas docentes en la enseñanza universitaria. Revista de Docencia Universitaria. REDU. Monográfico: Buenas prácticas docente en la enseñanza universitaria, 10 (1), 17-42. Recuperado de http://goo.gl/sY1TAf 


\section{Para citar este artículo}

Fernández Alex, M.D. (2016). Modelo educativo emergente en las buenas prácticas TIC. Revista Fuentes, 18(1), 33-47. [Fecha de consulta: dd/mm/aa]. doi: doi: http://dx.doi.org/10.12795/revistafuentes.201 6.18.1.02 